\title{
Impronta del espacio público en la formación del ser humano
}

\author{
Marcela Otárola Guevara
}

Artículo

Invitada nacional

Profesional independiente

E-mail: motarolag@gmail.com

Recibido: 1 de junio de 2018

Aprobado: 6 de diciembre de 2018

\section{Marcela Otárola Guevara}

Costarricense, arquitecta, profesional independiente, Magister Scientiae en Historia Aplicada de la Universidad Nacional (2015) y doctoranda del programa de Doctorado en Estudios de la Sociedad y la Cultura de la Universidad de Costa Rica.

\section{Resumen}

El crecimiento progresivo y acelerado de las urbes en las últimas décadas, ha producido asentamientos cada vez más densos donde convergen seres humanos con distintas procedencias que se apropian del espacio público, fáctica o simbólicamente. Reflexionar cómo repercute esta transformación de la morfología urbana en la conciencia de quien habita la ciudad, requiere establecer un derrotero teórico que facilite, en primera instancia, la comprensión de los procesos de construcción de sí mismo que realiza el sujeto y que le permite interpretar y vincularse con su entorno y todo lo que él contiene. Para lograr este cometido, se retoman propuestas teóricas que vienen de distintos campos cognoscentes que muestran varias formas de forjar la subjetividad del individuo, evidencian la importancia del rol del espacio como lugar de socialización y permiten elucidar mecanismos de desigualdad que atentan contra la convivencia humana.

Palabras clave: subjetividad; intersubjetividad; espacio público; desigualdad.

\section{Public space imprint on human being's education}

\begin{abstract}
The progressive and accelerated growth of the cities in the last decades, has produced increasingly dense settlements where human beings converge with different origins that appropriate the public space, factually or symbolically. Reflecting how this transformation of urban morphology affects the consciousness of those who inhabit the city, requires establishing a theoretical course that facilitates, in the first instance, the understanding of the processes of self-construction that the subject performs and that allows him to interpret and bond with their environment and everything that it contains. To achieve this goal, theoretical proposals that come from different knowledge fields that show various ways of forging the subjectivity of the individual, show the importance of the role of space as a place of socialization and allow to elucidate mechanisms of inequality that attempt against human coexistence.
\end{abstract}

Keywords: subjectivity; intersubjectivity; public space; inequality. 


\section{Preámbulo}

Las urbes cambian como resultado de la acción del individuo y éste, a la vez, es transformado por su espacio circundante, lo que revela su incidencia en la subjetividad e intersubjetividad humana. Al considerar esta situación, y con el fin de aprovechar aportes desde distintos ámbitos epistémicos, en este escrito se acopian premisas que advierten cómo operan en el espacio procesos de elaboración de conciencia humana y cómo afectan en el sentido y orden dado al territorio.

Para iniciar este ejercicio, se parte de la definición de subjetividad e intersubjetividad usando como referencia, nociones que proceden de la fenomenología social para introducir el tema de la construcción social de la realidad basada en la interacción humana. Esta visión se complementa con las contribuciones de investigadoras quienes, desde el feminismo y con el afán de desmantelarlas, estudian lógicas de dominio que determinan al sujeto para subyugarlo.

Seguidamente, se procede a espacializar los procesos de producción de la subjetividad y, para ello, se establece el espacio público como el lugar relacional donde se construye el individuo, razón por la que adquiere distintas connotaciones: como lugar donde habita la palabra para evidenciar el proceso de diálogo mediante el cual se genera el conocimiento que interioriza en su cosmovisión; y como lugar donde converge la diferencia por la confluencia de grupos heterogéneos que han sido discriminados en otros sitios y no han compartido procesos de cohesión que facilite su convivencia.

La falta de conexión humana produce lugares de encuentro con un débil tejido social, circunstancia que se denuncia al hablar de espacios públicos muertos para referirse a zonas comunales donde la convivencia ha sido menoscabada. Para comprender este deterioro, se requiere analizar los dispositivos de desigualdad que acontecen en los espacios públicos y que se revelan en la disposición del uso del suelo y la estigmatización basada en imaginarios, lo cual ha dado como resultado espacios proscriptos que congregan sujetos divergentes.

\section{Una aproximación a la subjetividad e intersubjetividad como procesos de vinculación}

Para ilustrar la pertinencia del estudio de la subjetividad e intersubjetividad en reflexiones de naturaleza espacial, se comparte algunos datos sobre las transformaciones de los asentamientos humanos en el siglo actual.

Según la organización Hábitat para la Humanidad (2014) en el año 2014, las ciudades albergaron dos terceras partes de la población urbana mundial. Cuatro años antes, de acuerdo con un informe del Banco Mundial (2016), la región de América Latina y el Caribe registró un $80 \%$ de esa población, Centroamérica un $56.88 \%$ y, en el caso específico de Costa Rica un $71.73 \%$ con una proyección de alcanzar el $90 \%$ en el 2050.

Vislumbrando este panorama, casi todos los habitantes costarricenses vivirán en el siglo XXI en urbes y es allí donde surgen las siguientes interrogantes: ¿cómo se conformarán esos espacios de coincidencia humana? ¿Cómo se relacionarán quienes los frecuentan y habitan? ¿Cómo afectarán la conciencia de sus usuarios?

Un dato más, en el año 2010 el índice de aglomeración en Costa Rica (indicador que relaciona la densidad poblacional en $1 \mathrm{~km} 2$ con el acceso a una ciudad mayor a 50.000 habitantes) fue 53\% (Banco Mundial, 2016, p. 10), es decir, más de la mitad de los costarricenses vivían cerca de las ciudades de la Gran Área Metropolitana cuyos poblados, recientemente, se han extendido producto de migraciones internas en un movimiento centrífugo -del centro a la periferia- (Instituto Nacional de Estadística y Censos, 2014), lo que ha provocado modificaciones en la estructura demográfica de zonas suburbanas (regiones extrarradio de localidades consolidadas).

Ante este escenario, los espacios urbanos costarricenses se han tornado en "el mundo de la vida cotidiana," noción del sociólogo y filósofo Alfred Schütz, y retomada por la investigadora en comunicación Marta Rizo (2005) para llamar al sitio de la acción social 
donde convergen personas con diferentes trayectorias sociales (Alvarez Sousa, 1996) quienes, al interaccionar, establecen valores y procesos de interpretación compartidos (Rizo, 2005, p.6) que otorgan sentido a una realidad. De esta forma, se infiere que estos espacios de confluencia son también lugares donde coexiste la heterogeneidad y están atravesados por distintas discursividades instauradoras de procesos de subjetivación e intersubjetivación que afectan al individuo.

Por subjetivación se entiende el conocimiento que, a partir de una perspectiva propia y holística, tiene el sujeto del entorno y de sí mismo y que es compartido y replanteado cotidianamente al interactuar con otros, intercambio que se denomina intersubjetividad (Rizo, 2005).

Estudios sobre el feminismo que tratan las relaciones de poder exponen este proceso desde un enfoque donde la definición del sujeto está estrechamente ligada a lo que la filósofa María Luisa Femenías (2000) llama lógicas de dominio y que intervienen en la formación de la identidad, conceptualización que se aprecia cuando habla del concepto poscolonial de contra-identidad para señalar que la visión de mundo del colonizado está influenciada por su colonizador:

Si como bien afirman algunas teóricas postcoloniales, surge en los colonizados un fenómeno inevitable que denominan contra-identidad, que se moldea en la experiencia de la colonización, nada permite inferir que esta contra-identidad no se construya a partir de parámetros patriarcales agiornados y modelizados según las coordenadas del caso. (Femenías, 2000, p. 249)

Otra investigadora, la antropóloga Rosalva Aida Hernández Castillo (2008) presenta un ejemplo similar al citar a la escritora Gloria Alzandúa para robustecer su cuestionamiento sobre la creación de identidades monolíticas, estáticas y excluyentes, que no corresponden a una realidad contemporánea en la que conviven sujetos disímiles y complejos en constante proceso de reformulación personal:

Como feminista, Alzandúa se rebela ante el machismo del nacionalismo chicano y ante sus definiciones limitadas y «disciplinarias» de la tradición [...] No se propone hacer una teoría general de la identidad ni plantear que las identidades siempre se viven como múltiples y contradictorias, simplemente da cuenta de que en el nuevo contexto global, hay muchos sujetos que, como ella, vivimos nuestras identidades como un amasamiento, y a los que nos «zumba la cabeza con lo contradictorio». (Hernández Castillo, 2008, p. 82)

Por otro lado, la socióloga e historiadora Silvia Rivera Cusicanqui (2010), en oposición a los intelectuales latinoamericanos que formulan un tipo de colonialismo fundamentado en un "nuevo canon académico" y a los que considera defraudadores por apropiarse de las contribuciones de otros eruditos en la construcción del conocimiento, emplea el concepto de ch'ixi para diferenciarse de ellos (a quienes llama q'ara: usurpadores de lo ajeno) y también para definirse como persona y destacar la mezcla que subyace en el individuo:

Por eso, me considero ch'ixi, y considero a ésta la traducción más adecuada de la mezcla abigarrada que somos las y los llamados mestizas y mestizos. La palabra ch'ixi tiene diversas connotaciones: es un color producto de la yuxtaposición, en pequeños puntos 0 manchas, de dos colores opuestos o contrastados: el blanco y el negro, el rojo y el verde, etc. Es ese gris jaspeado resultante de la mezcla imperceptible del blanco y el negro, que se confunden para la percepción sin nunca mezclarse del todo. (Rivera, 2010, p. 69)

En los ejemplos supra citados se elucidan, principalmente, la intersubjetividad y su incidencia en la construcción de la subjetividad del ser humano. En términos identitarios, todas coinciden en que la subjetividad revela las consecuencias de los vínculos que establece un ser humano con sus similares: para Femenías se evidencia en las creencias del colonizador que, renovadas para vencer cualquier resistencia, se insertan en la cosmovisión del ser subalterno; para Hernández Castillo la intersubjetividad se proyecta en la complejidad del sujeto, fruto de su interacción con los múltiples y constantes estímulos que recibe del entorno mientras que para Rivera Cusicanqui deviene de la historia mestiza del individuo que acarrea las diferencias culturales que lo definen. 


\section{El espacio de la subjetividad e intersubjetividad}

Ahora bien, entendidas la subjetividad y la intersubjetividad como un proceso indivisible que se sustenta en la interacción humana es primordial reflexionar sobre el espacio donde se llevan a cabo, especialmente en el ámbito público, porque se considera es el sitio donde se generan la mayor cantidad de relaciones que inciden en la constitución del ser humano. No obstante, antes de continuar con este abordaje, es necesario aclarar que el ámbito privado es una espacialidad muy relevante en la formulación de la subjetividad de la mujer y el hombre, pero el interés de este trabajo se enfoca en lo público por su correspondencia con asuntos urbanos que comportan una socialización más amplia del individuo.

Bajo esta perspectiva, y con el afán de mostrar su presencia la de las interacciones en un espacio más perceptible (como el urbano), se trae a colación la noción de lo público que establece el sociólogo Richard Sennett (1978) escrita en su libro El declive del hombre público y que define al explicar los términos de lo «público» y lo «privado» para, posteriormente, visualizarlos en las relaciones humanas acontecidas en el ámbito urbano:

...lo «público» viene a significar una vida que transcurre fuera de la vida, de la familia y de los amigos cercanos. En la región pública, los grupos sociales complejos, distintos, habrían de llegar a un contacto indefectible. La ciudad capital constituía el foco de esta vida pública. (Sennett, 1978, p. 27)

Tal concepto es desarrollado por la geógrafa social Patricia Ramírez Kuri (2003) quien, en sus investigaciones sobre el tema de la ciudad y la vida pública, sitúa el dominio público en un espacio compartido donde la persona construye su realidad y a sí misma mediante la socialización:

En este lugar común, que se usa o puede usarse con propósitos públicos o privados, la gente se relaciona con su entorno físico y social, se encuentra con la historia propia o la de otros, en escenarios que conjugan elementos naturales, socioculturales y arquitectónicos con prácticas sociales que pueden estimular o limitar la comunicación y la integración social entre individuos y grupos diferentes. (Ramírez, 2003, p. 37)

El contacto indefectible mencionado por Sennett y el espacio relacional al que refiere Ramírez Kuri aluden a zonas de reciprocidad donde prima la interacción y la percepción como actividades fundamentales para la formación de lo que Rizo (2005) llama sujeto social, individuo quien en esas áreas dialoga con su conciencia y las de sus convecinos para establecer una interacción donde se construye a sí mismo y aprehende al "otro" y así determina, en ambos casos, la existencia de un ser a partir de la comparación entre sujetos (p. 5).

El reconocimiento del otro está influido por condiciones que constriñen la subjetividad y para ello es necesario contextualizar este acto en un medio intervenido por prácticas políticas, económicas y simbólicas que se denomina espacio público, mismo que Ramírez Kuri precisa en una alocución sobre la Ciudad de México:

... en los espacios públicos urbanos confluyen usos, significados y funciones diversos y heterogéneos que hacen visibles los problemas que plantea la vida pública [...] Formas plurales de expresión, convivencia, descanso, recreación, celebración, consumo y trabajo, se alternan con prácticas ciudadanas en demanda de reivindicaciones políticas y sociales. (Ramírez, 2003, p. 38)Las actividades descritas conllevan a la consideración del espacio público como la palestra donde el ser humano trasciende la satisfacción de sus necesidades de reproducción y supervivencia, pues en él se conforma la comunidad política, término que Armelle Le Bras-Chopard (2003) introduce al reseñar la construcción de lo que llama el "cuerpo político" en la Antigua Grecia, y en el que "... se lleva a cabo la humanidad del hombre" (p. 91).

Le Bras-Chorpad (2013) reflexiona, desde una perspectiva filosófica, sobre la naturaleza política del individuo y acude a los aportes de pensadores griegos para, en primera instancia, diferenciar al ser humano del animal, destacando la necesidad y la voluntad del hombre de convivir en comunidad y de la injerencia del espacio en la satisfacción de esa demanda. De este modo, define el cuerpo político como un cuerpo colectivo no biológico donde cada sujeto desarrolla su individualidad y se vincula a otros (p. 89) 
actos que ocurren en la ciudad, lugar conceptualizado como el medio donde el hombre y la mujer se realizan como tales: "El hombre es parte de la ciudad, no como un ser parcial semejante a su vecino, sino porque en su seno hace realidad su humanidad" (Le Bras-Chopard, 2003, p. 98).

En esta premisa se deja entrever que el cuerpo político no es una conjunción de personas, sino un tejido de individuos que logran identificarse como sujetos, es decir, constituye una entidad donde ocurre un proceso simultáneo de personalización y socialización gracias a la capacidad del hombre para relacionarse (Le Bras-Chopard, 2003, p. 99).

De esta vinculación social es pertinente destacar lo alusivo a la diferencia entre los seres humanos y su habilidad para entrelazarse, porque ello consigna el sitio que habilita estas dinámicas: el espacio público o la ciudad (en el sentido propuesto por Le Bras-Chopard) es lugar de la palabra (2003, p. 96) y lugar común de la diferencia (Ramírez, 2003, p. 35).

\section{El espacio público como el lugar de la palabra}

Hannah Arendt (citada por Le Bras-Chopard, 2003), establece el lugar de la palabra en la ciudad, no obstante, se considera oportuno sustituir el término ciudad por espacio porque éste es más amplio (no excluye lugares de socialización no urbanos) y alude a entornos que son socialmente construidos.

El espacio público habilita la interacción que pone a dialogar distintas subjetividades mediante el habla, principal medio de comunicación porque implica la visión y la escucha, acciones indiscutibles de relación (Rizo, 2005, p. 4), y que ha promovido la conversación como gesto para tratar temas trascendentales para el ser humano alejándolo de la "conducta de los animales y los bárbaros" (Le Bras-Chopard, 2003, p. 97).

Marta Rizo (2005) asevera que la transmisión visual y oral como forma primordial de comunicación forja una realidad social común que moldea la subjetividad de los individuos dado que el diálogo favorece no sólo la expresión sino también la interpretación, lo que otorga a la persona una dualidad que Eduardo Vizer, autor aludido por Rizo, define como "la doble posición de actores y observadores"(p. 4). En este sentido, el desempeño de la persona como actor cobra relevancia por cuanto se apunta a que el sujeto reconoce y asume la práctica de roles, entendidos éstos como conductas predeterminadas por ciertas circunstancias que llevan intrínsecas creencias, mismas que Richard Sennett señala como el resultado de la aprehensión de una ideología que se proyecta en el comportamiento humano (Sennett, 1978, p. 48).

De acuerdo con lo planteado por este estudioso, se infiere que los roles son proyecciones de dogmas condicionados socialmente y, en el espacio público, son importantes porque ellos median en las relaciones que generan cohesión en la población para evitar el desconocimiento entre individuos que debilita los entramados sociales.

Cuando lo extraño o ajeno impera en la interacción, las actuaciones humanas en la sociedad son para Sennett (1978) similares a los papeles que asume un actor de teatro: en ambas situaciones la carencia de conocimiento sobre el otro impide comprender su conducta, de alli que se procede a creer, solamente, en lo que dice su comportamiento sin indagar en todo aquello que la origina.

Este tema remite al concepto de performance que dicta Judith Butler con respecto al género. Butler (2007) afirma que el género es uno de varios "estilos de la carne", deviene de una historia que lo condiciona y es un acto en el tanto se ejerce para experimentar y legitimar significados acopiados socialmente. El género requiere de un exterior donde visibilizar la "ilusión de un yo constante" que no está sujeto a una identidad sustancial, sino que procede de una construcción social del cuerpo (p. 274).

De acuerdo con lo anterior, se deduce que el performance es una representación del individuo que requiere de un espacio para exponerse pues, al igual que el actor de Sennett, carga con la construcción de una historia (o varias) que es encarnada y pretende expresión. Ambos casos revelan que los seres humanos hablan de sí mediante 
sus obras y poseen un cuerpo que es simbólico, está traspasado por ideologías y permeado de la conciencia de los otros en quienes busca ser reconocido e interpretado y, por esta razón, demanda de un sitio para manifestarse: el espacio público, al que se le considera, por consiguiente, un lugar de enunciación del discurso, del ejercicio de la palabra como acción por excelencia para el entendimiento.

\section{El espacio público como lugar común de la diferencia}

El lugar común de la diferencia es una noción desarrollada por Patricia Ramírez Kuri (2003) a partir del análisis de la lectura que Richard Sennett hace de la ciudad moderna como una zona donde cohabitan la diferencia y la indiferencia, consecuencia de los cambios en la morfología urbana iniciados en la segunda mitad del siglo XX (p. 36) y que han provocado la densificación y diversificación de los asentamientos humanos, implicando la coexistencia de distintas personas sin una vinculación previa entre sí.

Tradicionalmente, el espacio público ha sido el espacio de encuentro de las personas donde se interactúa, se convive y se acuerda un orden que da sentido a la existencia individual y colectiva, sin embargo, con los procesos de urbanización devenidos de la Modernidad, se incrementó la densidad poblacional en las ciudades y ha proliferado la privatización de los espacios de encuentro por la creciente construcción de sitios exclusivos para la socialización (malls, parques de entretenimiento particulares y complejos habitacionales con amenities).

Quienes no pueden acceder a esos espacios privados convergen en los espacios públicos, mismos que se han tornado en puntos de convergencia de diferentes colectivos quienes "... con derechos y obligaciones diferenciadas en el entorno urbano, usan los lugares por motivos distintos -en unos casos comunes y en otros contrapuestos-..." (Ramírez, 2003, p. 37).

La apropiación que estas colectividades efectúan del espacio público provoca tiranteces y conflictividades por cuanto no se han establecido vasos comunicantes entre ellas para lograr su conocimiento y comprensión. En estos casos, por las diferentes causas que propiciaron la reunión de los grupos, éstos no configuran el tejido social que articula el cuerpo político que menciona Armelle Le Bras-Chopard (2003) pues no han sido participes de procesos de cohesión, sino por el contrario, han sido relegados allí por la exclusión; estos fueron diferenciados y segregados, fundamentalmente por su limitado poder adquisitivo (condición que está relacionada con otros factores como la etnia y el estatus migratorio por citar dos ejemplos).

Bajo esta perspectiva, la diferencia se conceptualiza conforme la acepción de Ana María Fernández (2009), quien afirma que lo diferente contraviene lo señalado hegemónicamente como lo Uno (referente a la uniformidad del ser) y por tanto es negativo e inferior. Quienes son señalados como diferentes son violentados mediante dispositivos de desigualación, mecanismos que Fernández identifica como procesos de marginalización, discriminación, exclusión y represión, que tienen asidero en la subjetividad (del hegemón y del subalterno) y se basa en una premisa fundamental simbólica: "diferente=inferior, peligroso o enfermo" (p. 37). Se vislumbra, entonces, que en el espacio público conviven sujetos que han sido víctimas de relaciones de poder centradas en el dominio sobre el otro (p. 48) negándoles derechos materiales y simbólicos como parte del sometimiento que padecen.

Diferenciar a un individuo alude a la conceptualización simbólica del cuerpo que realiza David Le Breton (2002) y no a su consideración como una realidad en sí misma y es mediante la estigmatización y desigualación cómo se segrega esta corporalidad. Excluir el cuerpo diferente evoca la idea del cuerpo individual de Le Breton, pues su visualización como una entidad que pertenece a una estructura social individualista sin nexos solidarios coincide con el sujeto ideal que los grupos de poder desean controlar y a quien, mediante la expoliación de sus bienes y potencias, condenan a una existencia en desventaja y desigualdad de condiciones (p. 22).

Así, al espacializar la diferencia, los espacios públicos elucidan los procesos de desigualación que imperan en la ciudad. La construcción de la diferencia que primó 
en la Antigüedad y que estuvo vigente por varios siglos, antes de la Modernidad, continuó con la diferenciación, no del cuerpo humano en búsqueda de su humanidad (comprendida como realización del ser), sino con la distinción y estigmatización de todo aquello que representa lo negativo de lo idéntico y amenaza un orden hegemónico, situación que presenta al lugar común de la diferencia como espejo de los distintos mecanismos de segregación social.

\section{El espacio público muerto}

El espacio público como lugar de expresión y reconocimiento que aglutina grupos segregados es común en países que padecen de un ordenamiento territorial dirigido por empresas inmobiliarias que optimizan la riqueza, sea por medio de la especulación de la tierra o por la construcción de grandes centros de consumo y se imponen a un Estado con un débil desempeño en gestiones urbanas.

La ciudad difusa o de baja densidad, esquema territorial planteando por esas compañías, disgrega asentamientos, desliga actividades y genera dependencia de vehículos automotores para realizar los recorridos, lo que suscita la atomización de contingentes humanos carentes de apego socio territorial. La persona que es parte de esta dinámica está desvinculada socialmente y, en este aspecto, se asemeja al hombre cosmopolita descrito por Sennett (1978) como aquel "...que se mueve cómodamente en la diversidad, se encuentra cómodo en situaciones que no tienen vínculo o paralelo con aquello que le es familiar"(p.27).

Este retrato del hombre cosmopolita remite a la narración sobre el nacimiento del individuo de David Le Breton y en ambos autores se encuentran dos coincidencias importantes: al igual que en el Renacimiento italiano, el comercio tiene una injerencia importante en el orden social y la figura del individuo moderno, que antepone el interés personal al bien común (Le Breton, 2002, p. 29) también se hace presente.

Así, la proliferación de sujetos devenidos de una individualización y de la dispersión urbana debilitan las redes sociales en tanto que los espacios que las han habilitado y contenido empiezan a carecer de sentido, pues el individuo ya no se inquieta por su comunidad, ni por las tradiciones ni creencias que comporta (Le Breton, 2002, p. 39), convirtiéndose dichos sitios en espacios públicos muertos (Sennett, 1978, p. 21) y por tanto susceptibles a controlar para perpetuar la heteronomía que favorece el statu quo de grupos de poder.

La circunstancia expuesta que tiende a presentarse en nuestro país (y que no es muy lejana a lo que acontece en otros sitios de América Latina) plantea el reto de encontrar la forma de devolverle a los espacios públicos su vocación de encuentro y, así, contribuir con la construcción de una subjetividad sustentada en la solidaridad (valor esencial para la vinculación social) y que impulse la libertad del ser humano. Para asumir el desafío señalado, inicialmente, es necesario reconocer que el espacio público es un territorio de discrepancia y ello conduce a esbozar las razones que lo precisan así.

\section{El espacio público como lugar de la divergencia}

Si los espacios públicos están en peligro de morir y, así, dejan de fomentar la reciprocidad que sustenta los procesos de subjetivación de una persona y su integración a un tejido social, ¿qué hacer? La interrogante sugiere hacer una revisión de los dispositivos de desigualación que afectan los espacios públicos urbanos.

En Costa Rica, los dispositivos de desigualación se expresan en las formas de exclusión relacionadas con el uso del suelo y en las significaciones imaginarias (concepto de Ana María Fernández que se entiende como un marco simbólico para la interpretación) de los pobladores de un territorio.

El uso del suelo es administrado por los gobiernos (locales y central) y está asociado a actividades que satisfacen las necesidades de quienes lo habitan y frecuentan. Actualmente, cambios demográficos en las ciudades de la Gran Área Metropolitana, 
demandan de una mayor cantidad y diversidad de servicios e infraestructura, modificando la morfología urbana y las distintas redes que la componen, labor que las municipalidades desarrollan al atender, especialmente, requerimientos del mercado inmobiliario.

Cambios de uso de suelo en barrios tradicionalmente habitacionales para fomentar la actividad comercial (que causa la expulsión de los residentes), el aumento y la concentración de construcciones residenciales inversamente proporcional al crecimiento de espacios públicos de recreación y socialización, así como la nula inversión en carreteras y servicios urbanos en zonas marginales revelan una violencia institucional (Fernández, 2009, p. 35) expresada en el manejo injusto de la dotación de recursos de infraestructura y de la administración del territorio, negando así el acceso a espacios y servicios de buena calidad a un grupo de ciudadanos.

Estos pobladores son de menor ingreso económico o están en condiciones de indigencia, condición que les impide integrarse al mercado de consumo y que los señala como "consumidores fallidos", término que emplea Zygmunt Bauman (2007) para definir a los pobres: personas ubicadas fuera de la norma porque no consumen, de allí que, no son comercializables, son inútiles y, por ende, deben ser invisibilizadas (p. 168).

Para legitimar su discriminación, los pobres a los que apunta Bauman son estigmatizados $\mathrm{y}$, consecuentemente, los lugares donde habitan o frecuentan son degradados por grupos en condición de privilegio, situación que se revela claramente en los medios de comunicación de circulación masiva: "Policías siembran terror en La Carpio" (Méndez, 2017), "Vecinos de Pavas se tratan de "cochinos" por tirar basura en la calle" (Castro, 2017) y "25.000 pobladores de Los Guido están condenados a vivir como precaristas" (Mata, 2014). La asociación de términos como terror, cochinos y precaristas con nombres de comunidades socialmente vulnerables como La Carpio, Pavas y Los Guidos, son un ejemplo de la puesta en práctica de las significaciones imaginarias para desigualar (Fernández, 2009, p. 41) que producen aislamiento espacial.

La incidencia de esta problemática en los espacios públicos se manifiesta en el debilitamiento de las redes sociales barriales y en la ralentización para crear unas nuevas, así como en la fragmentación de la composición social de las poblaciones.

Las modificaciones en el uso de suelo promueven el cambio de los usuarios de los espacios públicos, se marchan los antiguos, quienes heredaron y preservaron creencias y tradiciones arraigadas en el "mundo de la vida cotidiana" y arriban los nuevos que se asemejan al hombre cosmopolita por su desarraigo socio espacial. Así, las experiencias de vida se transforman, los procesos de comunicación se interrumpen y la interacción humana se trastoca instaurándose nuevos procesos de subjetivación, altamente orientados al individualismo.

En cuanto a las significaciones imaginarias, éstas favorecen la calificación peyorativa de comunidades que se traslada a sus habitantes, impidiéndoles por ello su acceso a otros espacios de socialización (particularmente aquellos de consumo y de carácter privado). Por esta razón, los espacios públicos se presentan a colectividades estigmatizadas como única alternativa para interactuar $y$, dado que cada vez esos sitios son menos, suelen confluir y generar tensiones debido a las apropiaciones que cada uno hace del lugar.

Así, espacios con rotación de usuarios por el cambio en las actividades económicas, políticas y sociales que condicionan nuevos usos del suelo y la reunión de grupos disímiles en busca de sitios para socializar congregan a personas con pensamientos discordantes, de allí la provocación a transformar los espacios públicos en lugar de coexistencia de la divergencia.

\section{A manera de cierre}

El ejercicio de poner a dialogar nociones de distintas disciplinas sobre la subjetividad e intersubjetividad ha permitido visibilizar las lógicas de dominio que imperan en el espacio público. Los mecanismos de desigualación que establecen una definición canónica del sujeto son aplicados por grupos detentores del poder político y financiero 
para favorecer la fragmentación de la sociedad y, con ello, estimular el individualismo del sujeto.

El aislamiento de la persona facilita su control, por cuanto el conocimiento que acopia ya no procede del espacio relacional que le otorgaba una red social y con la cual conformaba el cuerpo político, proviene -más que como conocimiento, como instrucción- de hegemones que visualizan el espacio público como el sitio donde instaurar alocuciones que condicionan la acción humana, es decir, como lugares donde acontecen actos performativos que modelan la subjetividad del ser en función de sus intereses particulares.

Se visualiza, entonces, que la subjetividad y la intersubjetividad son procesos susceptibles de fomentar adoctrinamientos, además de las desigualaciones que provocan la segregación anteriormente mencionada, de allí la importancia de observar las intenciones y los procesos que las instituyen con especial atención y diligencia si se toma en consideración la tendencia a vivir en asentamientos cada vez más heterogéneos.

¿Cómo lidiar, sin discriminar, con la diferencia manifiesta en apropiaciones espaciales divergentes?, ¿cómo contener la privatización de los espacios públicos que diluye el tejido social? o, ¿cómo resguardar u ofrecer espacios de emancipación al individuo? Estas elucubraciones son, más que cuestionamientos, provocaciones que incitan a continuar y profundizar en el estudio de los factores y las circunstancias que modelan la cosmovisión del sujeto. Asimismo, también conducen a reposicionar al ser humano en un sitio preponderante en el abordaje de temas pertinentes a la habitabilidad de las urbes, pues de no conocer la diversidad de cuerpos que contiene, las inequidades que allí ocurren y el fomento de perfomatividades que perpetúan la dominación, estaremos desarrollando sociedades de co-existencia y no de convivencia.

\section{Referencias Bibliográficas}

Alvarez Sousa, A. (1996). El constructivismo estructuralista: la teoría de las clases sociales de Pierre Bourdieu. Reis, 75, 145-172. Recuperado de http://www.reis. cis.es/REIS/PDF/REIS_075_08.pdf.

Banco Mundial. (2016). Estudio de la urbanización en Centroamérica "Oportunidades de una Centroamérica Urbana. Recuperado de http://documentos.bancomundial. org/curated/es/406571468196193946/pdf/106268-REVISED-SPANISH-PUBLICP152713-Central-America-Urbanization-Review-Final-Output-SPANISH-2.pdf

Bauman, Z. (2007). Vida de consumo. México D.F., México: Fondo de Cultura Económica.

Butler, J. (2007). El género en disputa. El feminismo y la subversión de la identidad. Barcelona: Paidós.

Castro, B (2017, 23 de mayo) Vecinos de Pavas se tratan de "cochinos" por tirar basura en la calle. La Prensa Libre. Recuperado de https://www.laprensalibre.cr/Noticias/ detalle/112576/vecinos-de-pavas-se-tratan-de-cochinos-por-tirar-basura-en-lacalle

Femenías, M. L. (2000). Sobre sujeto y género. Lecturas feministas desde Beauvoir a Butler. Buenos Aires: Catálogos.

Fernández, A. M. (2009). Las lógicas sexuales: amor, política y violencias. Buenos Aires: Nueva Visión.

Hábitat para la Humanidad (2014, 7 de octubre). Hábitat para la Humanidad innova en la creación de modelos urbanos. [Comunicado de prensa]. Recuperado de https://www.habitat.org/lac-es/quienes-somos/noticias/2014-10-7-modelosurbanos.

Hernández Castillo, R. A. (2008). Feminismos poscoloniales: reflexiones desde el sur del Río Bravo. En L. Suárez Navaz y R. A. Hernández Castillo (Eds), Descolonizando el feminismo. Teorías y prácticas desde los márgenes (75-113). Madrid: Ediciones Cátedra. 
Instituto Nacional de Estadística y Censos (2014). Estadísticas demográficas. 20112025. Proyecciones distritales. Población total por grupos de edades, según provincia y cantón [Estimaciones y proyecciones de población]. Recuperado de http://www.inec.go.cr/poblacion/estimaciones-y-proyecciones-de-poblacion

Le Bras-Chopard, A. (2003). El Zoo de los filósofos. De la bestialización a la exclusión. Madrid: Santillana Ediciones Generales.

Le Breton, D. (2002). Antropología del cuerpo y modernidad. Buenos Aires: Nueva Visión.

Mata, E. (2014,14 de julio) 25.000 pobladores están condenados a vivir como precaristas. La Nación. Recuperado de https://www.nacion.com/el-pais/gobierno/25000-pobladores-de-los-guido-estan-condenados-a-vivir-como-precaristas/ SLO33SVMTRFAZCVRBP5UHJFUX4/story/

Méndez,A(2017, 3 de mayo) Policías siembran terror en La Carpio. La Extra. Recuperado de http://www.diarioextra.com/Noticia/detalle/331524/policias-siembran-terror-enla-carpio

Ramírez Kuri, P. (2003). El espacio público: ciudad y ciudadanía. De los conceptos a los problemas de la vida pública local. En P. Ramírez Kuri (Ed), Espacio público y reconstrucción de ciudadanía (31-58). México D.F.

Rivera Cusicanqui, S. (2010). Ch'ixinakax utxiwa. Una reflexión sobre prácticas y discursos descolonizadores. Buenos Aires: Tinta Limón.

Rizo, M. (2005). La Intersubjetividad como Eje Conceptual para pensar la Relación entre Comunicación, Subjetividad y Ciudad. Razón y Palabra, 10 (47), 1-9. Recuperado de http://www.redalyc.org/articulo.oa?id=199520655003.

Sennett, R. (1978). El declive del hombre público. Barcelona: Ediciones Península. 